\title{
Comparative Experimental and Computational Study of Monoalkyl Chain
}

Phosphatidylcholine-Containing Thermoresponsive Liposomes

Kleopatra Eleftheriou ${ }^{\mathrm{a}}$, Zili Sideratou ${ }^{\mathrm{a}}$, Angelos Thanassoulas ${ }^{\mathrm{b}}$, Athanasios Papakyriakou ${ }^{\mathrm{a}}$, Dimitris Tsiourvas ${ }^{\mathrm{a}, *}$

a Institute of Nanoscience and Nanotechology, NCSR “'Demokritos”, 15310 Aghia Paraskevi, Attiki, Greece

${ }^{\mathrm{b}}$ Institute of Nuclear \& Radiological Sciences and Technology, Energy \& Safety, NCSR “'Demokritos”, 15310 Aghia Paraskevi, Attiki, Greece

Supporting Information 
Time-depended $\mathrm{CF}$ release at $37{ }^{\circ} \mathrm{C}$
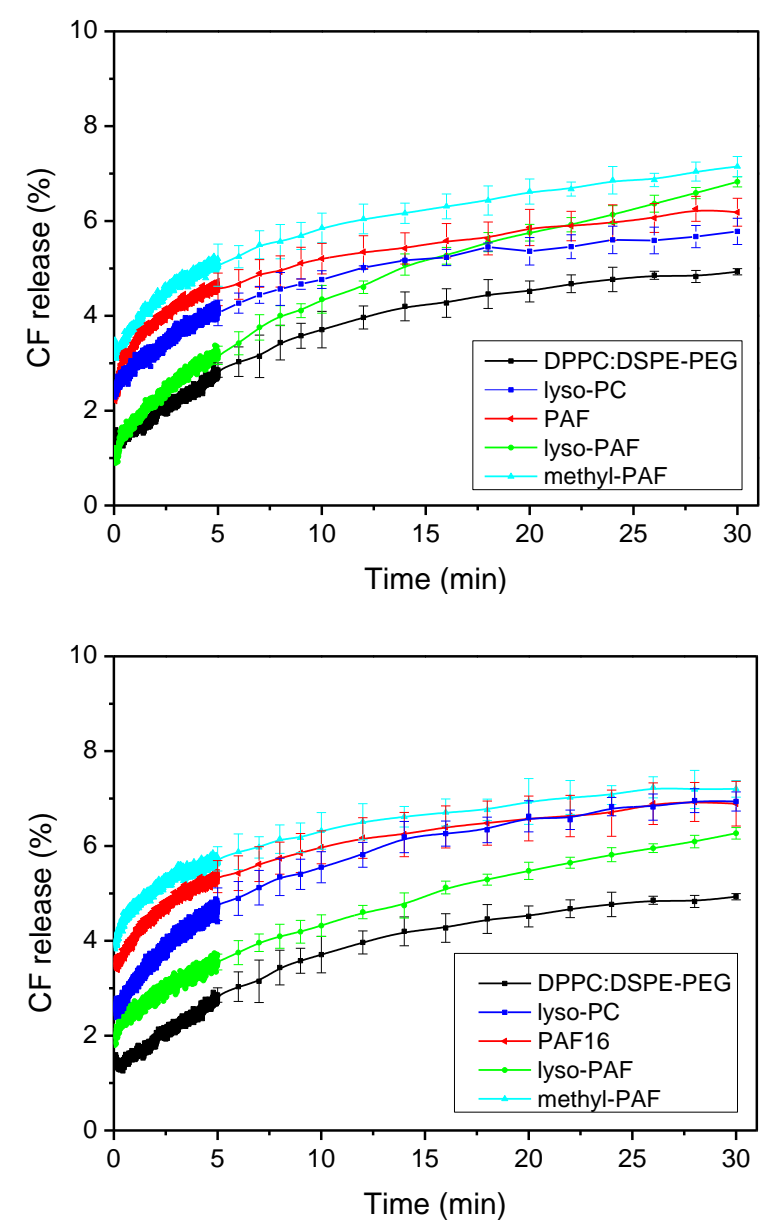

Fig. S1. Time dependent CF release at $37{ }^{\circ} \mathrm{C}$ from DPPC:DSPE-PEG based thermoresponsive unilamellar liposomes containing 5\% (upper part) or 10\% (lower part) of monoalkyl phospholipids. Measurements were made every $0.2 \mathrm{~s}$ during the first $5 \mathrm{~min}$ incubation time and every min afterwards. For clarity, error bars are inserted in the diagram after the first 5 min period. 
Table S1. CF release (\%) from liposomes after $24 \mathrm{~h}$ incubation at $37{ }^{\circ} \mathrm{C}$.

\begin{tabular}{lccc}
\hline Sample & & CF release (\%) & SE $( \pm)$ \\
\hline DPPC:DSPE-PEG & & 15.9 & 2.1 \\
\hline DPPC:DSPE-PEG:lyso-PC16 & $5 \%$ & 27.3 & 3.8 \\
DPPC:DSPE-PEG:PAF16 & $5 \%$ & 30.9 & 4.3 \\
DPPC:DSPE-PEG:lyso-PAF16 & $5 \%$ & 34.0 & 5.1 \\
DPPC:DSPE-PEG:methyl-PAF16 & $5 \%$ & 31.4 & 5.7 \\
\hline DPPC:DSPE-PEG:lyso-PC16 & $10 \%$ & 30.4 & 4.0 \\
DPPC:DSPE-PEG:PAF16 & $10 \%$ & 31.7 & 5.4 \\
$\begin{array}{l}\text { DPPC:DSPE-PEG:lyso-PAF16 } \\
\text { DPPC:DSPE-PEG:methyl-PAF16 }\end{array}$ & $10 \%$ & 34.7 & 4.2 \\
\hline
\end{tabular}




\section{Temperature and time-depended CF-release}

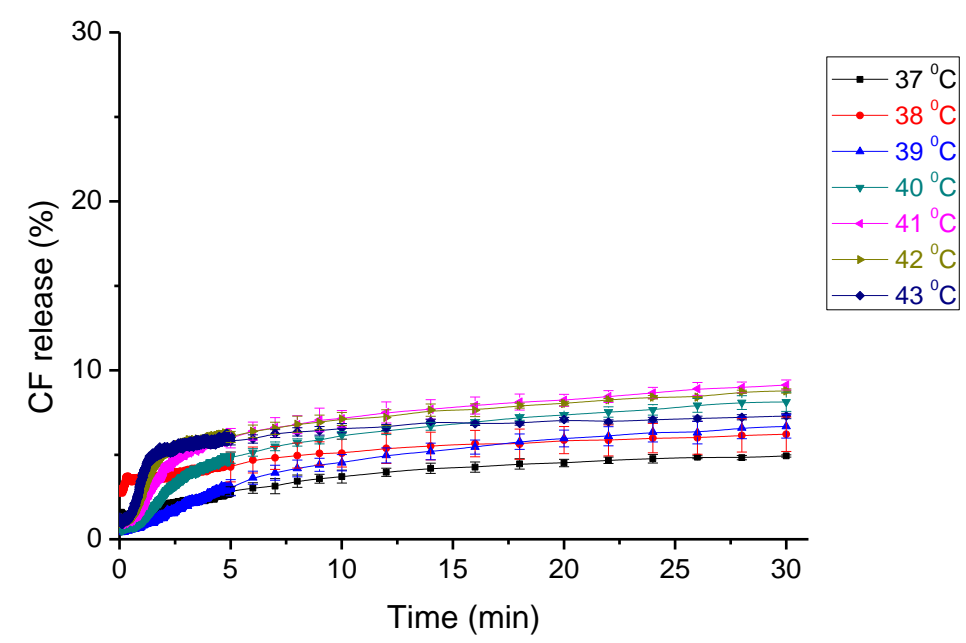

Fig. S2. Percent CF release vs. time from DPPC:DSPE-PEG (91:4) unilamellar liposomes at temperatures ranging from $37^{\circ} \mathrm{C}$ to $43^{\circ} \mathrm{C}$. Measurements were taken every $0.2 \mathrm{~s}$ during the first $5 \mathrm{~min}$ of incubation period, every $1 \mathrm{~min}$ until $10 \mathrm{~min}$, and every $2 \mathrm{~min}$ afterwards. For clarity, error bars are shown only after the first 5 min period. 


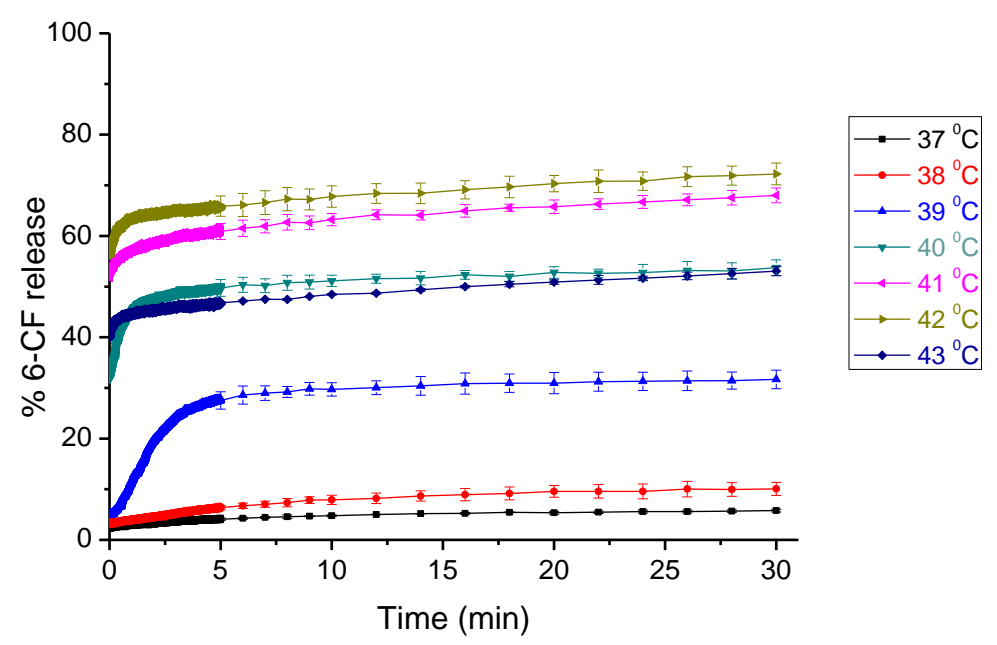

Fig. S3. Percent CF release vs. time from DPPC:DSPE-PEG:lyso-PC (91:4:5) unilamellar liposomes at temperatures ranging from $37^{\circ} \mathrm{C}$ to $43^{\circ} \mathrm{C}$.

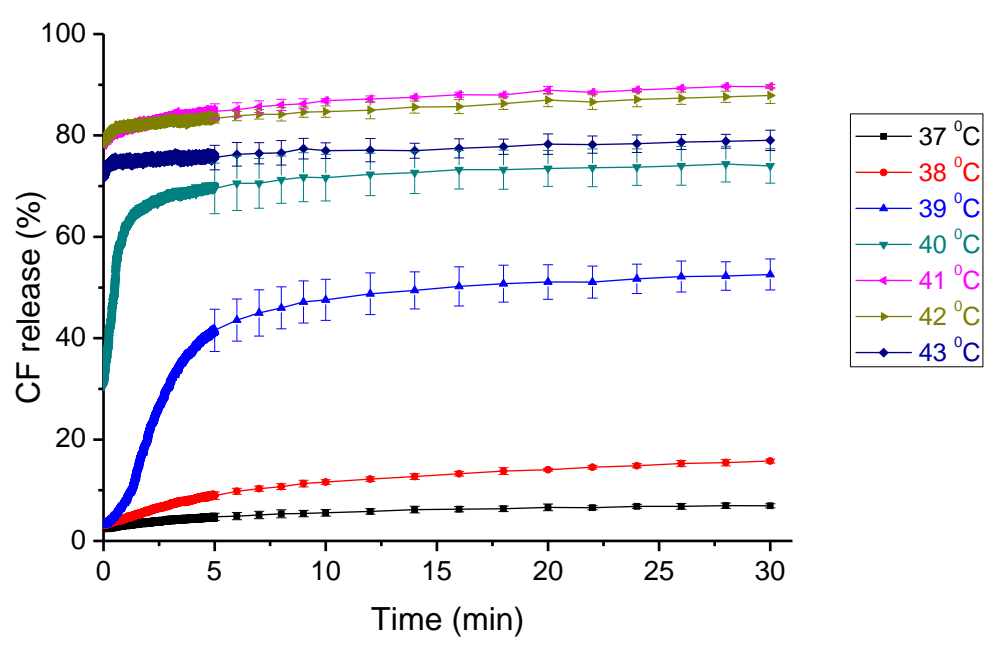

Fig. S4. Percent CF release vs. time from DPPC:DSPE-PEG:lyso-PC (91:4:10) unilamellar liposomes at temperatures ranging from $37^{\circ} \mathrm{C}$ to $43^{\circ} \mathrm{C}$. 


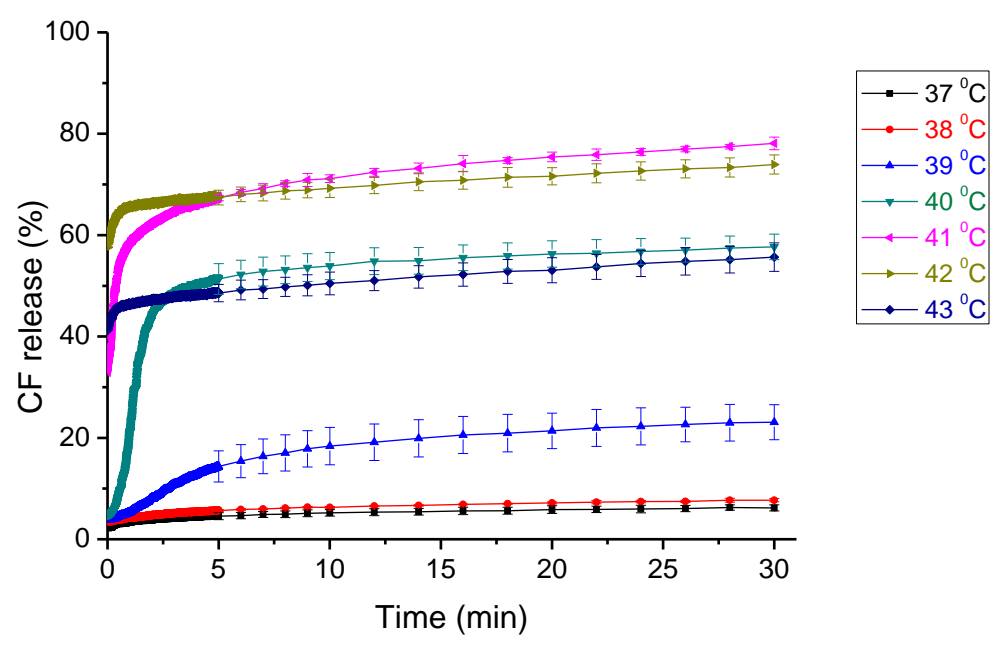

Fig. S5. Percent CF release vs. time from DPPC:DSPE-PEG:PAF (91:4:5) unilamellar liposomes at temperatures ranging from $37^{\circ} \mathrm{C}$ to $43^{\circ} \mathrm{C}$.

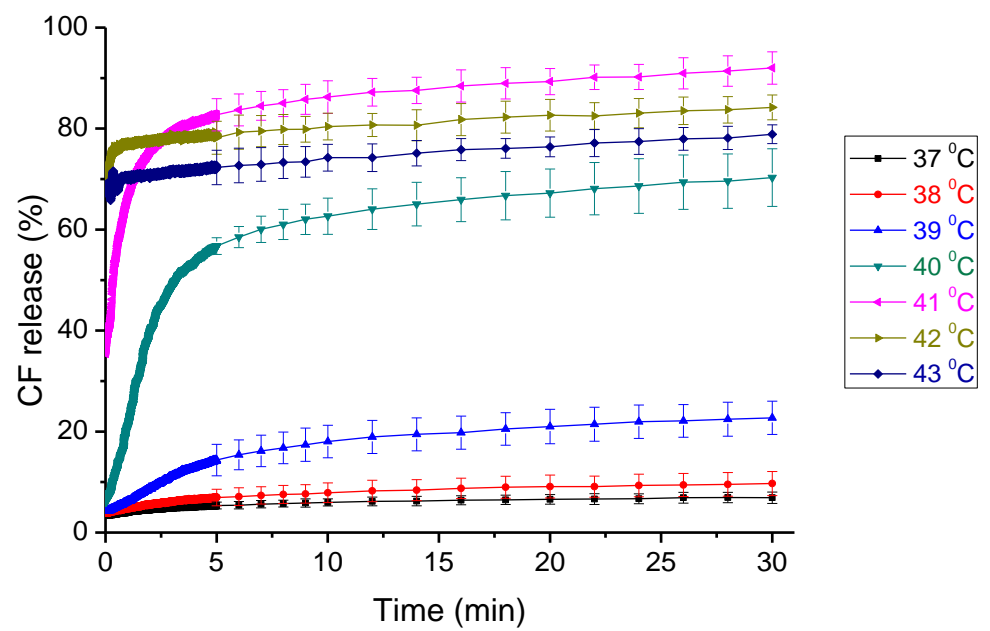

Fig. S6. Percent CF release vs. time from DPPC:DSPE-PEG:PAF (91:4:10) unilamellar liposomes at temperatures ranging from $37^{\circ} \mathrm{C}$ to $43^{\circ} \mathrm{C}$. 


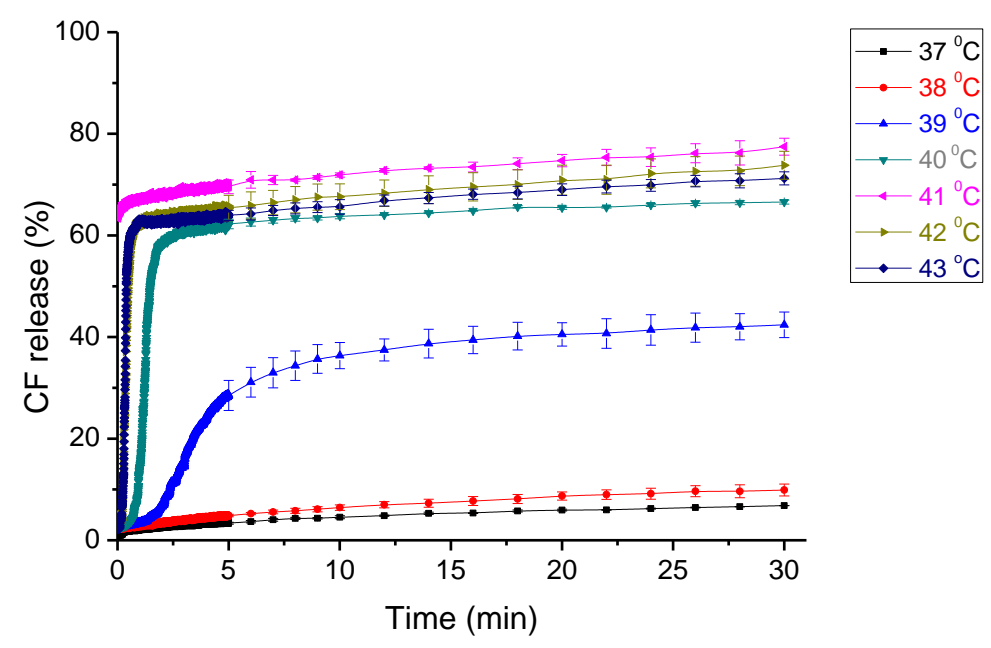

Fig. S7. Percent CF release vs. time from DPPC:DSPE-PEG:lyso-PAF (91:4:5) unilamellar liposomes at temperatures ranging from $37^{\circ} \mathrm{C}$ to $43^{\circ} \mathrm{C}$.

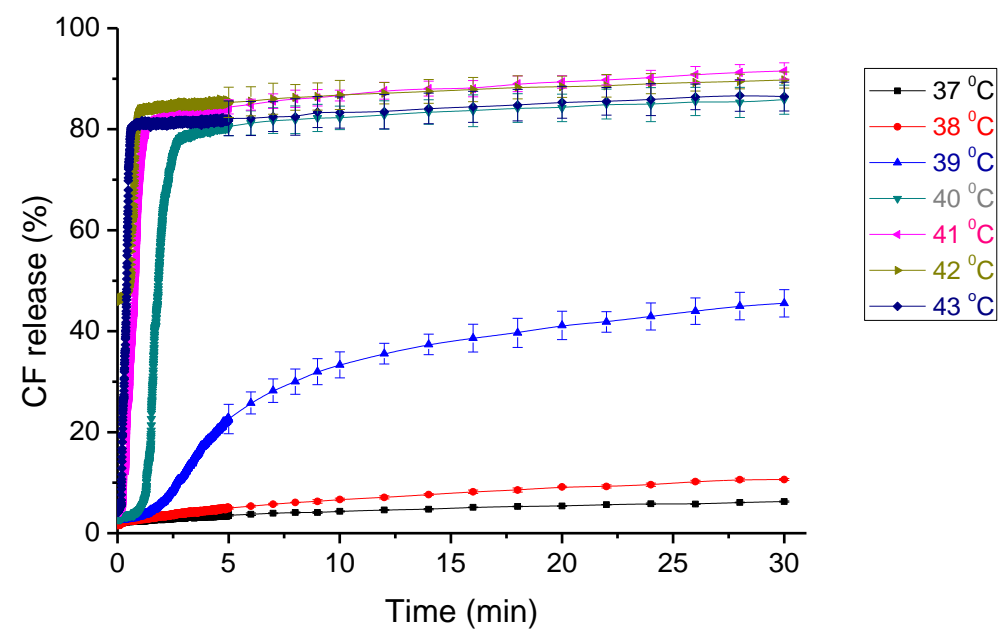

Fig. S8. Percent CF release vs. time from DPPC:DSPE-PEG:lyso-PAF (91:4:10) unilamellar liposomes at temperatures ranging from $37^{\circ} \mathrm{C}$ to $43^{\circ} \mathrm{C}$. 


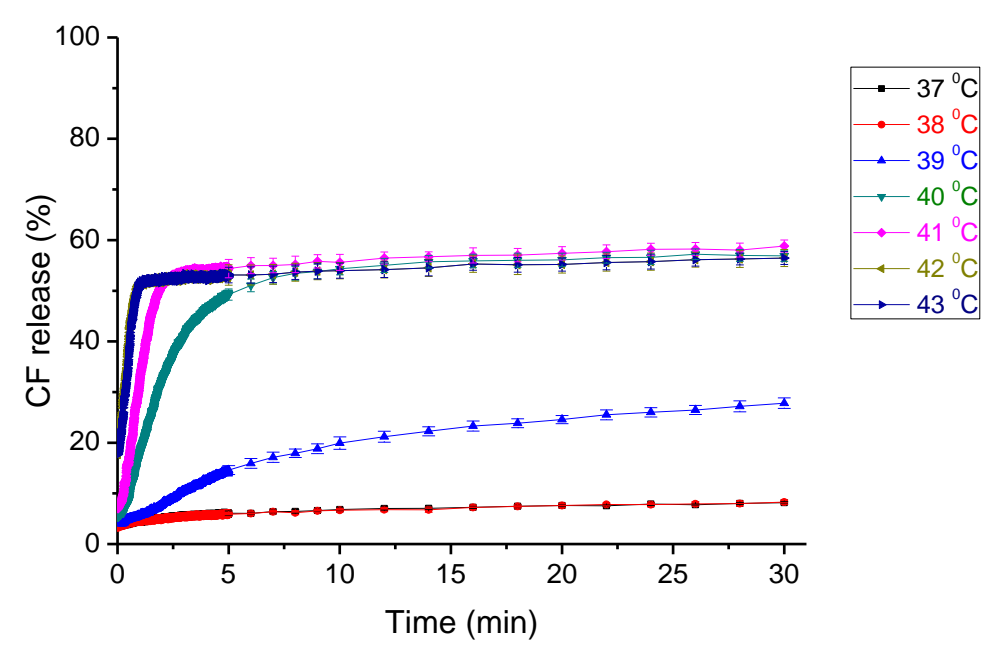

Fig. S9. Percent CF release vs. time from DPPC:DSPE-PEG:methyl-PAF (91:4:5) unilamellar liposomes at temperatures ranging from $37^{\circ} \mathrm{C}$ to $43^{\circ} \mathrm{C}$.

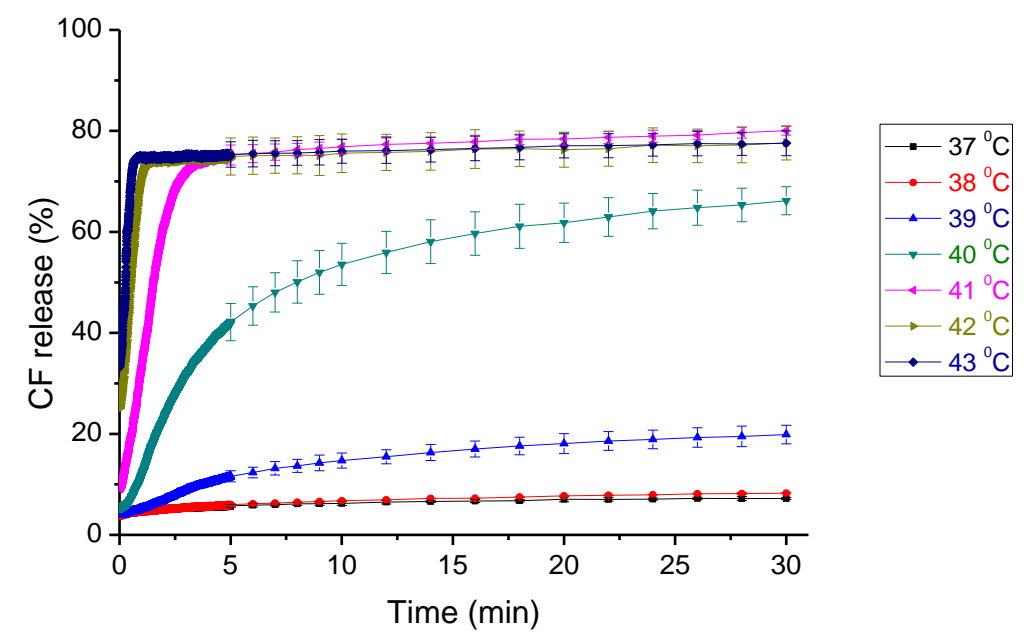

Fig. S10. Percent CF release vs. time from DPPC:DSPE-PEG:methyl-PAF (91:4:10) unilamellar liposomes at temperatures ranging from $37^{\circ} \mathrm{C}$ to $43^{\circ} \mathrm{C}$. 


\section{Computational Methods}

Force field parameterization. A new head group for each monoalkyl lipid was generated by mutating the phosphatidylcholine (PC) residue of the Lipid14 library, ${ }^{1}$ using the XLEaP module of AMBER $\mathrm{v} 14,{ }^{2}$ which would be suitable for building the monoalkyl lipid in combination with a single palmitoyl $\left(\mathrm{C}_{16}\right)$ acyl tail. Additional bonding parameters were taken from the General-AMBER force field (GAFF). ${ }^{3}$ Partial atomic charges were generated using the standard AMBER restraint electrostatic potential fit (RESP) ${ }^{4}$ protocol from optimization of the initial conformation and calculation of the ESP at the HF/6-31G* level using Gaussian 03. ${ }^{5}$ To further optimize the charge distribution of the lipids' headgroups, the four DPPCmonoalkyl lipid mixtures were equilibrated with 50-ns MD runs as described below. From the last $40 \mathrm{~ns}$ of each simulation, 40 randomly selected head-group conformations were extracted, were capped with a methyl group, and were used directly without optimization for calculation of the gas-phase ESP at the HF/6-31G* level. Partial charges were then calculated as an

\footnotetext{
${ }^{1}$ Dickson C. J.; Madej B. D.; Skjevik Å. A.; Betz R. M.; Teigen K.; Gould I. R.; Walker R. C. Lipid14: The Amber Lipid Force Field. J. Chem. Theory Comput. 2014, 10, 865-879.

${ }^{2}$ Case D. A.; Berryman J. T.; Betz R. M.; Cerutti D. S.; Cheatham T. E. III; Darden T. A.; Duke R. E.; Giese T. J.; Gohlke H.; Goetz A. W.; Homeyer N.; Izadi S.; Janowski P.; Kaus J.; Kovalenko A.; Lee T. S.; LeGrand S.; Li P.; Luchko T.; Luo R.; Madej B.; Merz K. M.; Monard G.; Needham P.; Nguyen H.; Nguyen H.T.; Omelyan I.; Onufriev A.; Roe D. R.; Roitberg A.; Salomon-Ferrer R.; Simmerling C. L.; Smith W.; Swails J.; Walker R. C.; Wang J.; Wolf R. M.; Wu X.; York D. M.; Kollman P. A. (2015), AMBER 2015, University of California, San Francisco.

${ }^{3}$ Wang J.; Wolf R. M.; Caldwell J. W.; Kollman P. A.; Case D. A. Development and testing of a general amber force field. J. Comput. Chem. 2004, 25, 1157-1174.

${ }^{4}$ Bayly C. I.; Cieplak P.; Cornell W.; Kollman P. A. A well-behaved electrostatic potential based method using charge restraints for deriving atomic charges: the RESP model. J. Phys. Chem. 1993, 97, 10269-10280.

${ }^{5}$ Gaussian 03, Revision D.01, M. J. Frisch, G. W. Trucks, H. B. Schlegel, G. E. Scuseria, M. A. Robb, J. R. Cheeseman, J. A. Montgomery, Jr., T. Vreven, K. N. Kudin, J. C. Burant, J. M. Millam, S. S. Iyengar, J. Tomasi, V. Barone, B. Mennucci, M. Cossi, G. Scalmani, N. Rega, G. A. Petersson, H. Nakatsuji, M. Hada, M. Ehara, K. Toyota, R. Fukuda, J. Hasegawa, M. Ishida, T. Nakajima, Y. Honda, O. Kitao, H. Nakai, M. Klene, X. Li, J. E. Knox, H. P. Hratchian, J. B. Cross, V. Bakken, C. Adamo, J. Jaramillo, R. Gomperts, R. E. Stratmann, O. Yazyev, A. J. Austin, R. Cammi, C. Pomelli, J. W. Ochterski, P. Y. Ayala, K. Morokuma, G. A. Voth, P. Salvador, J. J. Dannenberg, V. G. Zakrzewski, S. Dapprich, A. D. Daniels, M. C. Strain, O. Farkas, D. K. Malick, A. D. Rabuck, K. Raghavachari, J. B. Foresman, J. V. Ortiz, Q. Cui, A. G. Baboul, S. Clifford, J. Cioslowski, B. B. Stefanov, G. Liu, A. Liashenko, P. Piskorz, I. Komaromi, R. L. Martin, D. J. Fox, T. Keith, M. A. Al-Laham, C. Y. Peng, A. Nanayakkara, M. Challacombe, P. M. W. Gill, B. Johnson, W. Chen, M. W. Wong, C. Gonzalez, and J. A. Pople, Gaussian, Inc., Wallingford CT, 2004.
} 
average of the RESP-derived charges over all conformations. This approach has the advantage of producing Boltzmann weighted charges, while introducing a temperature dependence. ${ }^{1,6,7}$

Molecular dynamics equilibration. The initial structures were first minimized using 5000 steps of steepest descent and followed by 5000 steps of conjugate gradient minimization. Each system was then heated in two stages. In the first heating stage the system target temperature was slowly heated from 0 to $100 \mathrm{~K}$ over 5 ps with a constant volume simulation (NVT ensemble) using the Langevin thermostat with a collision frequency of $1.0 \mathrm{ps}^{-1} .8$ The second heating stage then increased the target temperature to $323 \mathrm{~K}$ over 1.0 -ns constant pressure simulation (NPT ensemble). The Langevin thermostat was used with the Berendsen barostat with a target pressure of 1.0 bar and a relaxation time of 1.0 ps. ${ }^{9}$ During the heating simulations, all atoms of the bilayer were restrained with a weak harmonic restraint to the initial structure using a force constant of $10 \mathrm{kcal} \mathrm{mol}^{-1} \AA^{-2}$ to prevent large structural deviations.

Molecular dynamics production runs. After heating, production dynamics was simulated with constant temperature and pressure with the Langevin thermostat at $323 \mathrm{~K}$ with collision frequency of $1.0 \mathrm{ps}^{-1}$, and the anisotropic Berendsen barostat with reference pressure of 1.0 bar and a relaxation time of 1.0 ps. The SHAKE algorithm ${ }^{10}$ was used to constrain bonds involving hydrogen with a relative tolerance of $1 \times 10^{-7}$. A time step of 2 fs was used in all stages of molecular dynamics. The particle mesh Ewald summation method was employed for long-range electrostatics and van der Waals interactions. ${ }^{11}$ The real space cut-off for the electrostatics was set to $10.0 \AA$ and a long-range dispersion correction was applied to the energy and pressure beyond this cut-off. For all bilayer systems lipid equilibration was

\footnotetext{
${ }^{6}$ Skjevik Å. A.; Madej B. D.; Walker R. C.; Teigen K. LIPID11: A modular framework for lipid simulations using Amber. J. Phys. Chem. B. 2012, 11, 11124-11136.

${ }^{7}$ Dickson C. J.; Rosso L.; Betz R. M.; Walker R. C.; Gould I. R. GAFFlipid: A general Amber force field for the accurate molecular dynamics simulation of phospholipid. Soft Matter 2012, 8, 9617-9627.

${ }^{8}$ Pastor R.; Brooks B.; Szabo A. An analysis of the accuracy of Langevin and molecular dynamics algorithms. Mol. Phys. 1988, 65, 1409-1419.

${ }^{9}$ Berendsen H. J. C.; Postma J. P. M.; van Gunsteren W. F.; DiNola A.; Haak J. R. Molecular dynamics with coupling to an external bath. J. Chem. Phys. 1984, 81, 3684-3690.

${ }^{10}$ Ryckaert J.-P.; Ciccotti G.; Berendsen H. J. C. Numerical integration of the cartesian equations of motion of a system with constraints: molecular dynamics of n-alkanes. J. Comput. Phys. 1977, 23, 327-341.

${ }^{11}$ Darden, T.; York D.; Pedersen L. Particle Mesh Ewald - an N.Log(N) Method for Ewald Sums in Large Systems. J Chem. Phys. 1993, 98, 10089-10092.
} 
performed for $50 \mathrm{~ns}$ in the NPT ensemble. Then, three independent NPT production runs of $200 \mathrm{~ns}$ for each bilayer were initiated from different random seeds (example of area per lipid versus simulation time graphs in Fig. S11).

Starting from the equilibrated systems at $323 \mathrm{~K}$, we performed three additional 200-ns NPT runs for DPPC and for each monoalkyl lipid-DPPC mixture at $314 \mathrm{~K}$. Each run was seeded from the last frame at $250 \mathrm{~ns}$ of the NPT simulations at $323 \mathrm{~K}$. While all four mixtures displayed equilibrated structural parameters, pure DPPC exhibited characteristics of the liquid to gel phase transition (e.g. the area per lipid as a function of simulation time shown in Fig. S11). The structural properties of pure DPPC at $314 \mathrm{~K}$ were equilibrated after $60-70 \mathrm{~ns}$, and for this reason the initial 100-ns steps of each NPT run at $314 \mathrm{~K}$ were not included in the averaging of the structural parameters, electron density, order parameters and diffusion calculations.
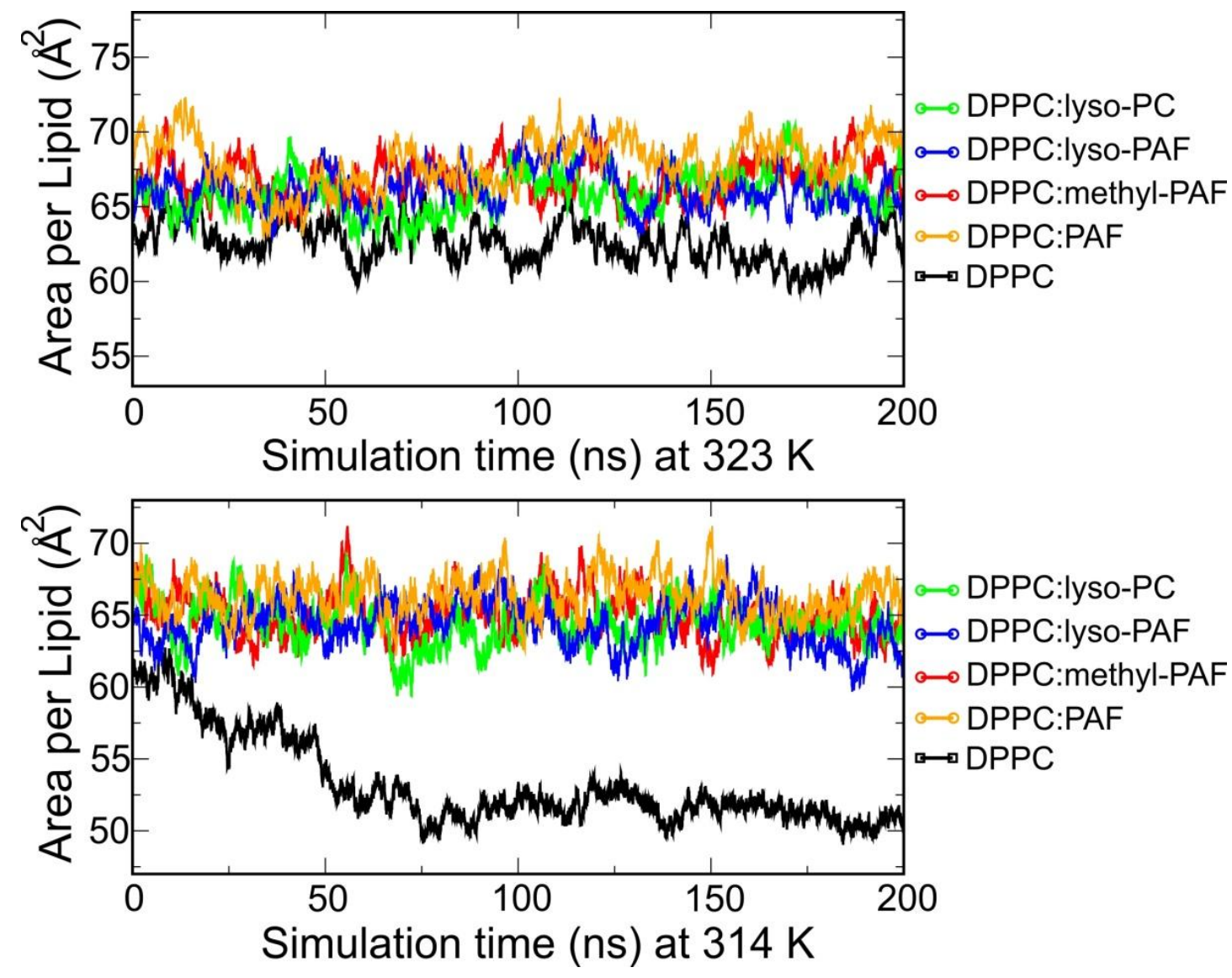

Fig, S11. Area per lipid as a function of simulation time from characteristic NPT simulations of DPPC (black) and the four DPPC:monoalkyl lipid systems at $323 \mathrm{~K}$ (upper) and $314 \mathrm{~K}$ (lower). Simulations at $314 \mathrm{~K}$ were seeded from the last frame of the equilibrated systems at $323 \mathrm{~K}$. 
Given that temperature regulation methods, such as Langevin dynamics that randomizes particle velocities, may affect dynamic properties such as diffusion, the lipid lateral diffusion was also determined from simulations in the microcanonical (NVE) ensemble. ${ }^{12}$ A single NVE production run of $100 \mathrm{~ns}$ for each lipid system was extended from the $200 \mathrm{~ns}$ NPT runs at $323 \mathrm{~K}$. To avoid energy and temperature drift, the time step was reduced to $1 \mathrm{fs}$, the center of mass motion was removed every 500 steps, and both the SHAKE and Ewald direct sum tolerance were made more stringent.

Structural and dynamic properties. Analysis of the trajectories was performed using the CPPTRAJ module of AmberTools v15 and custom R-based scripts. ${ }^{13}$ Results for the following parameters are given as block averages over the triplicate NPT simulations $(6 \times 100$ ns) \pm standard deviation (Tables 5 and 6 in the manuscript).

Area per lipid. The area per lipid $\left(A_{\mathrm{L}}\right)$ for each system was calculated using the dimensions of the simulation box according to:

$A_{L}=\frac{2 \times L_{x} \times L_{y}}{n_{\text {lipid }}}$

where $L_{\mathrm{x}}$ and $L_{\mathrm{y}}$ is the $\mathrm{x}$ - and $\mathrm{y}$-dimension of the simulation box, respectively, and $n_{\text {lipid }}$ is the number of lipids in the system.

Volume per lipid. The volume per lipid $\left(V_{\mathrm{L}}\right)$ was calculated using the dimensions of the simulation box according to:

$V_{L}=\frac{V_{\text {box }}-n_{W} V_{w}}{n_{\text {lipid }}}$

where $V_{\mathrm{box}}$ is the volume of the simulation box, $n_{\mathrm{W}}$ is the number of waters and $V_{\mathrm{W}}$ is the volume of each water molecule.

\footnotetext{
${ }^{12}$ Basconi J. E.; Shirts M. R. Effects of temperature control algorithms on transport properties and kinetics in molecular dynamics simulations. J. Chem. Theory Comput. 2013, 97, 2887-2899.

${ }^{13}$ Roe D. R.; Cheatham T. E. PTRAJ and CPPTRAJ: Software for processing and analysis of molecular dynamics trajectory data. J. Chem. Theory Comput. 2013, 97, 3084-3095.
} 
The volume of TIP3P water was estimated to be $31.1 \AA^{3}( \pm 0.1)$ from a 50-ns MD simulation of 1880 TIP3P molecules under the same experimental conditions as described for the NPT equilibration of the lipids. This value is in good agreement with the results obtained by analogues simulations in the literature (e.g. $\left.31.2 \AA^{3}\right){ }^{14}$

Bilayer thickness. The average thickness of the lipid bilayer $\left(h_{L}\right)$ was calculated as:

$h_{L}=\frac{V_{L}}{A_{L}}=\frac{V_{b o x}-n_{W} V_{w}}{2 \times L_{x} \times L_{y}}$

where $V_{\mathrm{L}}$ and $A_{\mathrm{L}}$ is the volume and area of the lipids, respectively, and $n_{\mathrm{W}}$ is the number of waters, $V_{\mathrm{W}}$ is the volume of each water molecule, $L_{\mathrm{x}}$ and $L_{\mathrm{y}}$ is the $\mathrm{x}$ - and $\mathrm{y}$-dimension of the simulation box, respectively. Bilayer head-to-head $\left(D_{\mathrm{HH}}\right)$ thickness was also calculated from electron density profiles of the equilibrated production runs by measuring the distance between the maxima of the phosphate groups. Bilayer thicknesses from each simulation were averaged and the mean values are given.

Isothermal compressibility moduli. The isothermal area compressibility modulus $\left(K_{\mathrm{A}}\right)$ was calculated from the fluctuation in the area per lipid via:

$K_{A}=\frac{2 k_{\mathrm{B}} T\left\langle A_{L}\right\rangle}{n_{\text {lipid }} \sigma_{A}^{2}}$

where $k_{\mathrm{B}}$ is Boltzmann's constant, $T$ is the temperature, $\left\langle A_{\mathrm{L}}\right\rangle$ is the mean area per lipid and $\sigma_{\mathrm{A}}{ }^{2}$ is the variance in the area per lipid. In general, $K_{\mathrm{A}}$ values are in good agreement with experimental values falling within the standard deviation.

Electron Density Profiles. The electron density profiles were calculated by assuming an electron charge equal to the atomic number minus the atomic partial charge, located at the center of each atom. Profiles have also been decomposed into contributions from the following groups: water (WAT), choline (CHL), phosphate (PO4), glycerol (GLY), carbonyl (COO), methylene (CH2), and terminal methyl groups (CH3). The profiles obtained at $323 \mathrm{~K}$ are all symmetrical, with water penetrating up to the carbonyl groups, leaving the terminal methyl groups dehydrated in agreement with experimental findings at $323 \mathrm{~K}$ (Fig. S12, S13).

\footnotetext{
${ }^{14}$ Hofsäß, C.; Lindahl, E.; Edholm, O. Molecular Dynamics Simulations of Phospholipid Bilayers with Cholesterol. Biophys. J. 2003, 84, 2192-2206.
} 
The electron density profiles extracted from the simulations of pure DPPC at $314 \mathrm{~K}$ (Fig. S14) revealed a liquid-to-gel phase transition, in contrast to those obtained for the four DPPC:monoalkyl lipid bilayers at $314 \mathrm{~K}$ (Fig. S15).

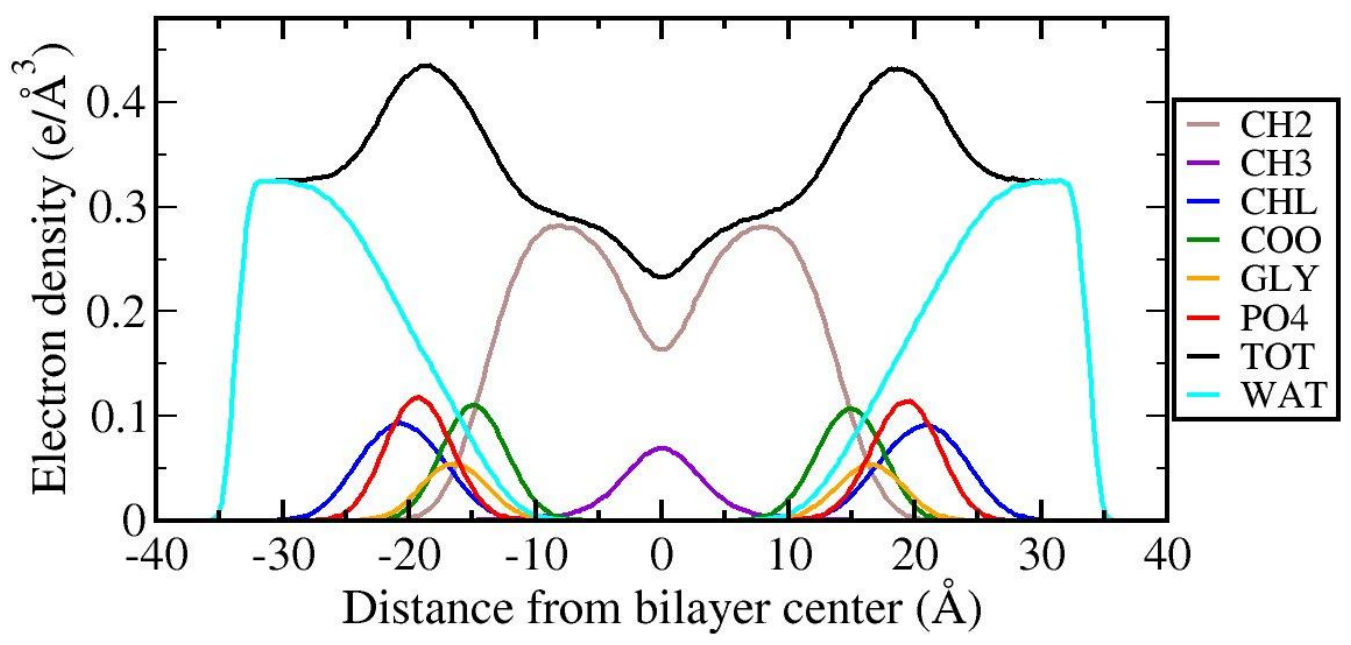

Fig. S12. The total (TOT) and decomposed electron density profile of DPPC lipid bilayer from the NPT simulations at $323 \mathrm{~K}$, with contributions from water (WAT), choline (CHOL), phosphate (PO4), glycerol (GLY), carbonyl (COO), methylene ( $\mathrm{CH} 2)$, and terminal methyl groups $(\mathrm{CH} 3)$.
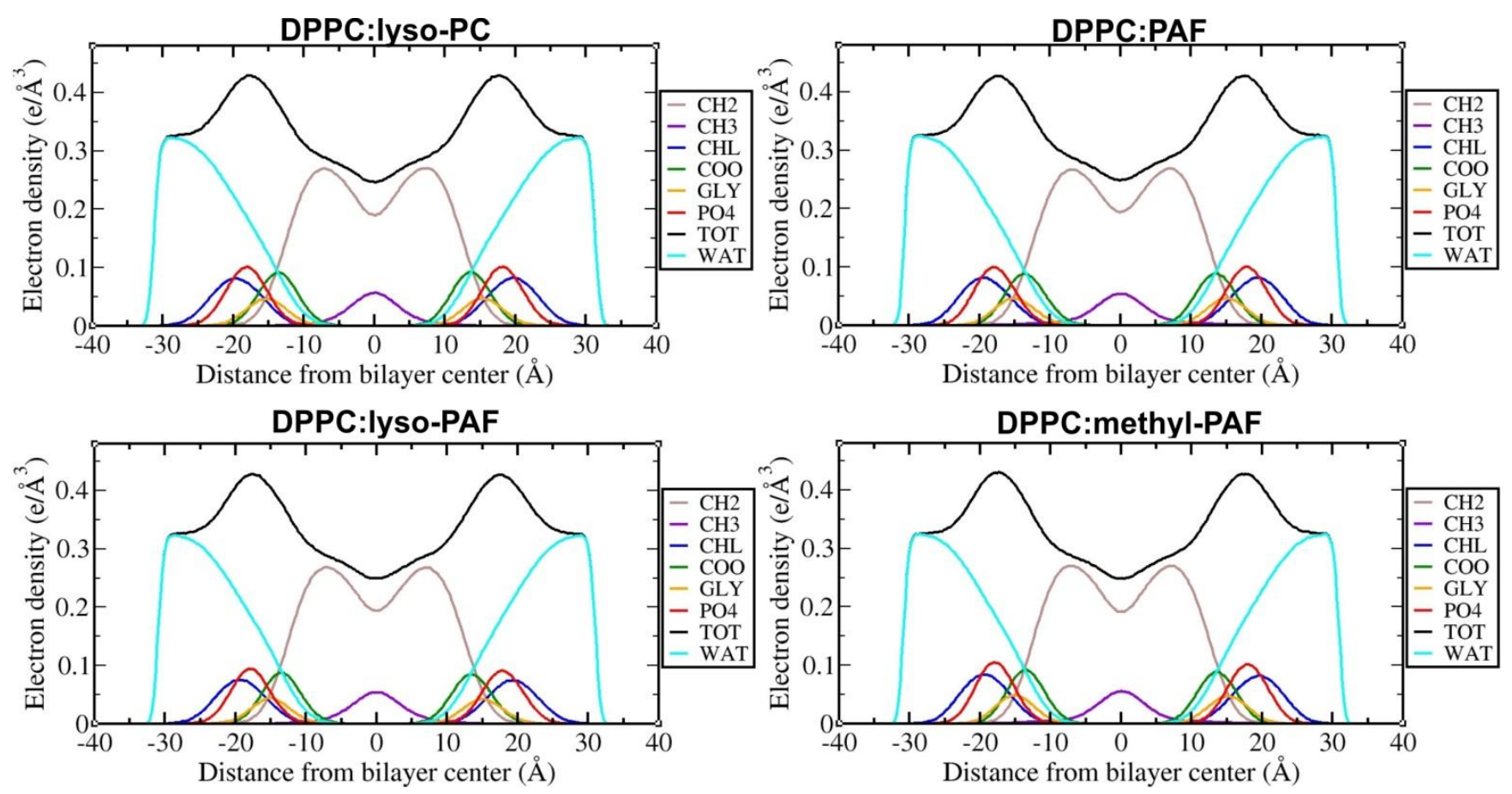

Fig. S13. The total and decomposed electron density profile of DPPC-monoalkyl lipid mixture systems extracted from NPT simulations at $323 \mathrm{~K}$ (color codes as in Fig. S12). 


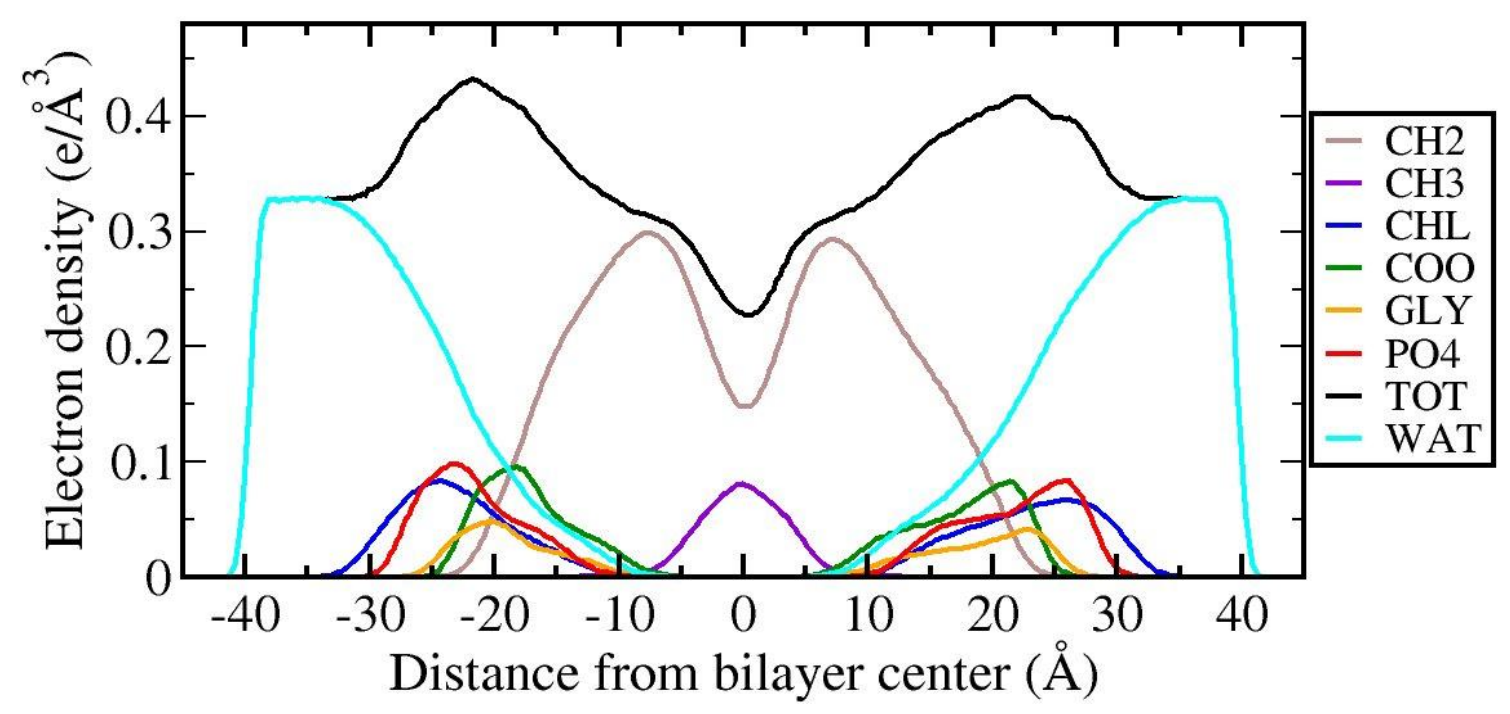

Fig. S14. The total (TOT) and decomposed electron density profiles of DPPC lipid bilayer from the NPT simulations at $314 \mathrm{~K}$, with contributions from water (WAT), choline (CHOL), phosphate (PO4), glycerol (GLY), carbonyl (COO), methylene (CH2), and terminal methyl groups $(\mathrm{CH} 3)$.
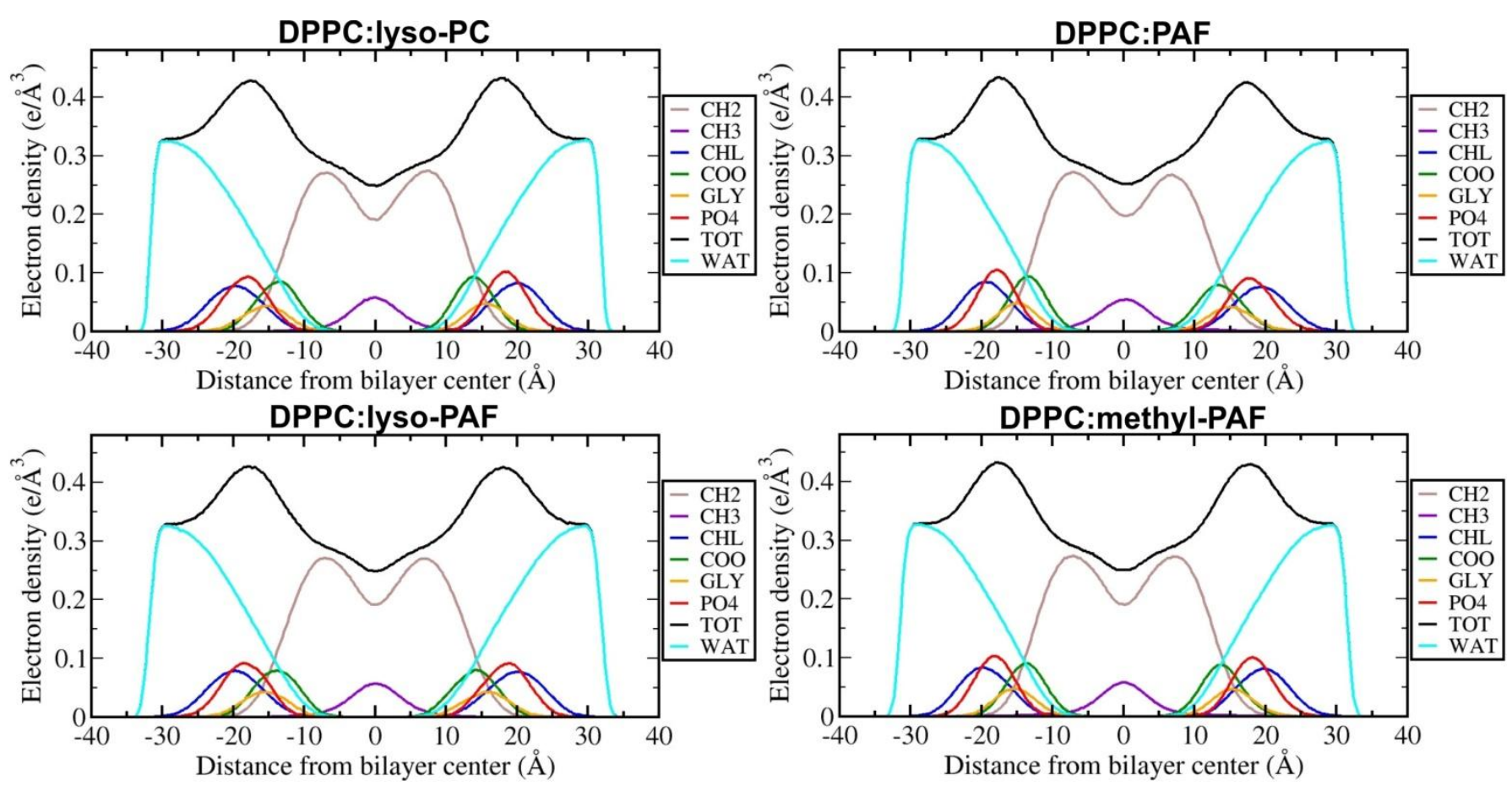

Fig. S15. The total and decomposed electron density profile of DPPC:monoalkyl lipid mixture systems extracted from the NPT simulations at $314 \mathrm{~K}$ (color codes as in Fig. S14). 
Ordering of Lipid Acyl Chains. NMR order parameters provide important information about glycerophospholipid acyl tail configurations. Experiments with ${ }^{2} \mathrm{H}$ NMR or ${ }^{1} \mathrm{H}-{ }^{13} \mathrm{C}$ NMR measure the splitting or coupling that is proportional to an order parameter value. The deuterium order parameter $S_{\mathrm{CD}}$ is a measure of the relative orientation of the C-D bonds with respect to the bilayer normal and is defined by the equation:

$$
\left|S_{\mathrm{CD}}\right|=\frac{1}{2}\left\langle 3 \cos ^{2} \theta_{\mathrm{CD}}-1\right\rangle
$$

where $\theta_{\mathrm{CD}}$ is the angle between the $\mathrm{C}-\mathrm{D}$ bond vector and the bilayer normal, and « > represents an ensemble average over all lipids.

$S_{\mathrm{CD}}$ values were calculated using the 'lipidorder' function in the CPPTRAJ module of AmberTools. ${ }^{13}$ The pure DPPC system at $323 \mathrm{~K}$ follows the experimental order parameter trend, as observed in the development of the Lipid14 force field. ${ }^{1}$ The $\mathrm{C}-2$ atoms of the $s n 1$ and $s n 2$ chains display markedly different order parameters owing to the different alignment of the acyl chains in this region. Experimental data indicate that $S_{\mathrm{CD}}$ order parameters of the $\mathrm{C}-\mathrm{D}$ bonds near the headgroup in the $s n 1$ chains are higher than the corresponding values of the $s n 2$ chains (Fig. S16). The simulations of pure DPPC at $314 \mathrm{~K}$ revealed a liquid-to-gel phase transition as evident by the significantly increased $\mathrm{S}_{\mathrm{CD}}$ values of DPPC chains up to C14 (Fig. S17). In contrast, the DPPC:monoalkyl lipids at $314 \mathrm{~K}$ displayed characteristic liquid phase $\mathrm{S}_{\mathrm{CD}}$ values, comparable to the results obtained at $323 \mathrm{~K}$ (Fig. S17).
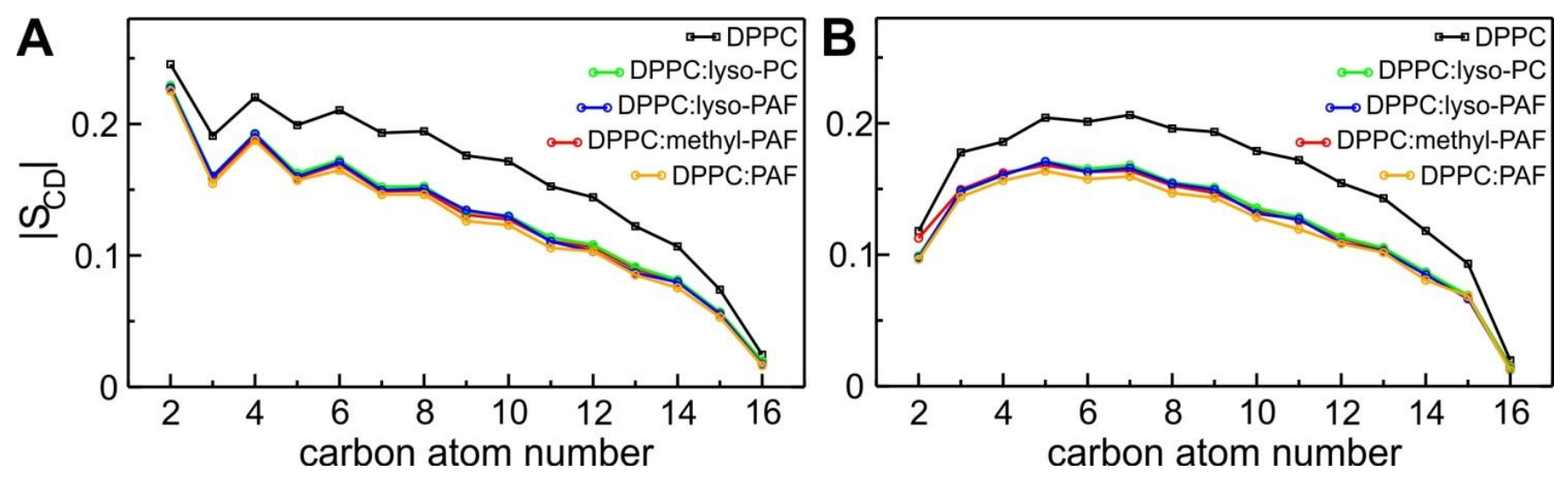

Fig. S16. $\left|\mathrm{S}_{\mathrm{CD}}\right|$ order parameter of DPPC acyl chains $s n 1$ (A) and $s n 2(\mathrm{~B})$ averaged over three independent 200-ns NPT simulations at $323 \mathrm{~K}$ for pure DDPC (black line) and DPPC in the presence of $10 \%$ molar fraction monoalkyl lipids. 

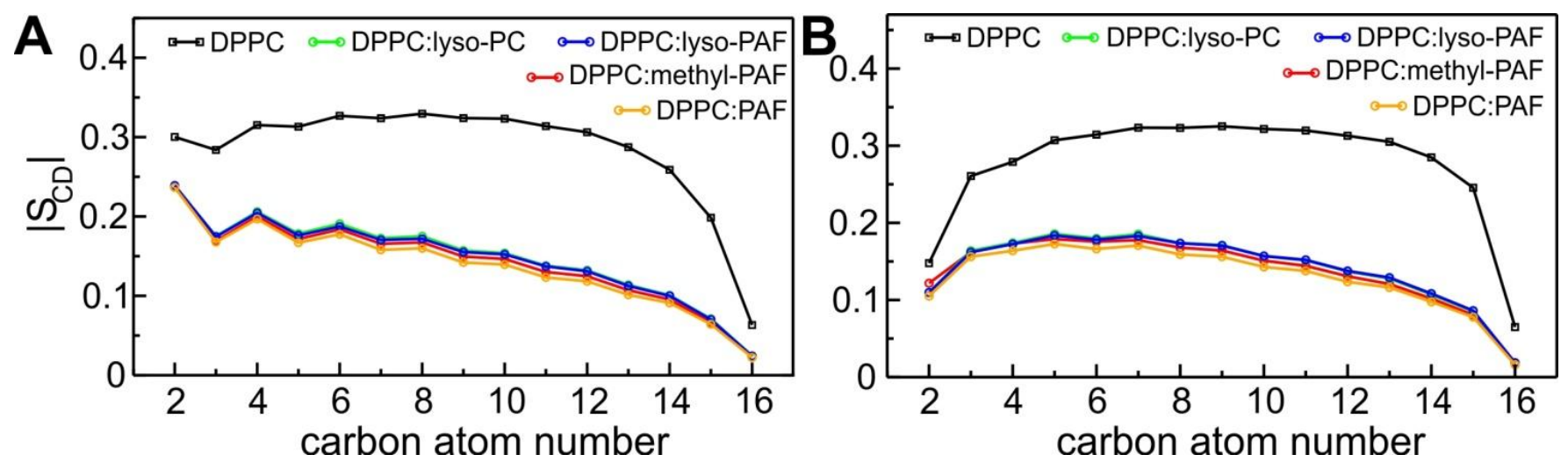

Fig. S17. $\left|\mathrm{S}_{\mathrm{CD}}\right|$ order parameter of DPPC acyl chains $s n 1$ (A) and $s n 2$ (B) averaged over three independent 200-ns NPT simulations at $314 \mathrm{~K}$ for pure DDPC (black line) and DPPC in the presence of $10 \%$ monoalkyl lipids.

Lipid lateral diffusion. The lateral diffusion coefficient $D_{\mathrm{xy}}$ for each lipid system was calculated using the slope of a mean-square displacement (MSD) plot versus time for the centers of mass, averaged over the trajectories of each molecule via the Einstein relation:

$D_{\mathrm{xy}}=\lim _{t \rightarrow \infty} \frac{\left\langle\Delta r(t)^{2}\right\rangle}{2 n_{i} t}$

where $\Delta r(t)$ is the distance that the lipid travels in time $t$ and $n_{\mathrm{i}}$ is the number of dimensions (for $D_{\mathrm{xy}}: n_{\mathrm{i}}=2$ ).

Diffusion coefficients were computed for each lipid as a block average of 100 ns over the three independent 200-ns NPT simulations. The mean-square displacement (MSD) curves were determined using window lengths spanning $20 \mathrm{~ns}$ and averaged over different time origins separated by 200 ps. The slope of this curve yields the diffusion coefficient using eq. 6, with the linear 10-20 ns region used to perform the fit. The lipid lateral diffusion was also calculated from a single 100-ns run in the NVE ensemble at $323 \mathrm{~K}$, as described above, with the time averaged MSD curves shown in the Fig. S18. The diffusion coefficients from the NVE runs are slightly higher than the $D_{\mathrm{xy}}$ values from the NPT runs as observed previously, ${ }^{1}$ albeit falling within the observed standard deviation of $0.5-1.5 \times 10^{-8} \mathrm{~cm}^{2} \mathrm{~s}^{-1}$ (Table S2). 


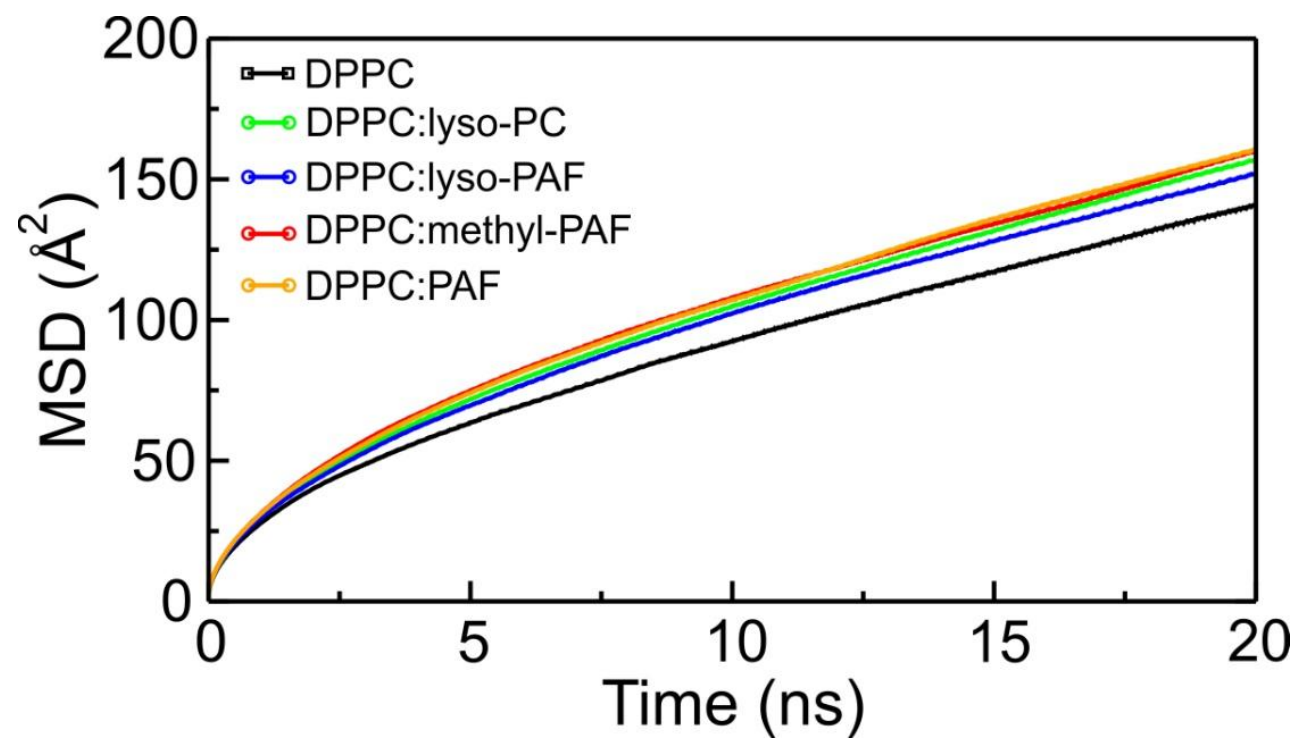

Fig, S18. Mean Square Displacement (MSD) curves of pure DPPC and the four DPPCmonoalkyl lipid $10 \%$ mol. systems from the NVE simulations at $323 \mathrm{~K}$.

Table S2. Lipid lateral diffusion coefficients calculated from three 200-ns NPT runs, a single 100-ns NVE run, and experimental values for pure DPPC and the four DPPC:monoalklyl lipid systems at $323 \mathrm{~K}$.

\begin{tabular}{|l|c|c|c|}
\hline Lipid system at 323 K & $\begin{array}{c}\mathbf{N P T} \boldsymbol{D}_{\mathbf{x y}} \\
\left(\mathbf{1 0}^{-8} \mathbf{c m}^{\mathbf{2}} \mathbf{~ s}^{-\mathbf{1}}\right)\end{array}$ & $\begin{array}{c}\mathbf{N V E} \boldsymbol{D}_{\mathbf{x y}} \\
\left(\mathbf{1 0}^{-\mathbf{8}} \mathbf{c m}^{\mathbf{2}} \mathbf{s}^{-\mathbf{1}}\right)\end{array}$ & $\begin{array}{c}\text { Experimental } \boldsymbol{D}_{\mathbf{x y}} \\
\left(\mathbf{1 0}^{-\mathbf{8}} \mathbf{c m}^{\mathbf{2}} \mathbf{~ s}^{\mathbf{- 1}}\right)\end{array}$ \\
\hline $\begin{array}{l}\text { Pure DPPC } \\
\text { 128 lipids }\end{array}$ & $11.0 \pm 0.7$ & $12.1 \pm 0.6$ & $12.5^{15}$ and $15.2^{16}$ \\
\hline DPPC:lyso-PC & $13.0 \pm 0.9$ & $13.1 \pm 0.7$ & - \\
\hline DPPC:PAF & $12.9 \pm 1.3$ & $13.4 \pm 1.2$ & - \\
\hline DPPC:lyso-PAF & $11.7 \pm 1.4$ & $12.4 \pm 0.7$ & - \\
\hline DPPC:methyl-PAF & $12.5 \pm 1.5$ & $13.0 \pm 0.5$ & - \\
\hline
\end{tabular}

\footnotetext{
15 Vaz W. L. C.; Clegg R. M.; Hallmann D. Translational diffusion of lipids in liquid crystalline phase phosphatidylcholine multibilayers. A comparison of experiment with theory. Biochemistry 1985, 24, 3781-786.

${ }^{16}$ Scheidt H. A.; Huster D.; Gawrisch K. Diffusion of cholesterol and its precursors in lipid membranes studied by ${ }^{1} \mathrm{H}$ pulsed field gradient magic angle spinning NMR. Biophys. J. 2005, 89, 2504-2512.
} 